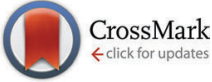

Cite this: Phys. Chem. Chem. Phys., $2016,18,6316$

Received 30th October 2015 Accepted 26th January 2016

DOI: $10.1039 / c 5 c p 06619 k$

www.rsc.org/pccp

\title{
Molecular structure and vibrations of NTCDA monolayers on Ag(111) from density-functional theory and infrared absorption spectroscopy $\dagger$
}

\author{
Ralf Tonner, ${ }^{\star a b}$ Phil Rosenow ${ }^{\mathrm{a}}$ and Peter Jakob*bc
}

\begin{abstract}
The structure and vibrational properties of the metal-organic interface of 1,4,5,8-naphthalene-tetracarboxylic dianhydride (NTCDA) on $\mathrm{Ag}(111)$ were analysed using Fourier-transform infrared absorption spectroscopy in conjunction with density functional theory calculations including dispersion forces (PBE-D3). Mode assignments and polarizations as well as molecular distortions were determined for four adsorption geometries of NTCDA on top and bridge sites aligned either parallel or perpendicular to the Ag rows and compared to accurate calculations of the free molecule. This enables an in-depth understanding of surface effects on the computed and experimental vibrational spectra of the adsorbed NTCDA molecule. The molecule-substrate interaction comprises two major and equally important contributions: non-directional van der Waals forces between molecule and surface, and covalent bonding of the acyl oxygen atoms with underlying Ag atoms, which is quantified by charge-transfer analysis. Furthermore, adsorption energy calculations showed that the molecular axis of flat-lying NTCDA is oriented preferably in parallel to the Ag rows. The molecule is subject to particular distortions from the planar gas phase structure with covalent bonding leading to downward bending of the acyl oxygen atoms and Pauli repulsion to upward bending of the carbon core. In parallel, strong buckling of the silver surface was identified. As found in previous studies, the lowest unoccupied molecular orbital (LUMO) of the molecule slips below the Fermi level and becomes partially populated upon adsorption. Excitation of totally symmetric vibrational modes then leads to substantial interfacial dynamical charge transfer, which is convincingly reproduced in the calculated IR spectra.
\end{abstract}

\section{Introduction}

Metal-organic interfaces represent key elements of optoelectronic devices like light emitting diodes or field effect transistors as the macroscopic device performance is often affected by structural and electronic properties at the atomic scale. The understanding of complex interfaces is, however, still limited and model systems are needed for further advancement of methods, which can subsequently be applied to more realistic interfaces. In general, the quality of organic films critically depends on the properties of the metal-organic interface and a comprehensive characterization

\footnotetext{
${ }^{a}$ Fachbereich Chemie, Philipps-Universität Marburg, 35032 Marburg, Germany. E-mail: tonner@chemie.uni-marburg.de

${ }^{b}$ Wissenschaftliches Zentrum für Materialwissenschaften (WZMW), Philipps-Universität Marburg, 35032 Marburg, Germany

${ }^{c}$ Fachbereich Physik, Philipps-Universität Marburg, 35032 Marburg, Germany. E-mail: peter.jakob@physik.uni-marburg.de

$\dagger$ Electronic supplementary information (ESI) available: Table on calculated structural data of adsorbed NTCDA; tables on vibrational frequencies of free and adsorbed NTCDA on Ag(111) (various adsorption sites); computed IR spectra of adsorbed NTCDA on $\mathrm{Ag}(111)$ for various adsorption sites; thermal evolution of low coverage NTCDA/Ag(111) IR spectra. See DOI: 10.1039/c5cp06619k
}

of the first organic layer on the metal substrate (contact primer layer) is of vital importance for an improved understanding of the growth kinetics of organic layers with well-defined properties. A profound understanding of internal interfaces may therefore be attained by analysing the first monolayer in detail. Preferentially, such studies are conducted using techniques which are likewise applicable to internal interfaces, such as infrared absorption spectroscopy (IRAS).

The interaction of large organic molecules with solid substrates at metal-organic interfaces comprises different contributions. ${ }^{1,2}$ Firstly, covalent bonds can be formed which are directional and closely related to the electronic structure of the molecule and the substrate, with particular emphasis on molecular orbitals located at or near the Fermi level of the substrate. Secondly, isotropic dispersion interactions are non-directional and site-insensitive and depend only on distance and polarizability of the atoms involved. In addition, characteristics of the molecular structure as well as the atomic arrangement and relaxations of the substrate atoms need to be taken into account. ${ }^{3}$ Aside from the moleculesubstrate interactions, lateral forces among adsorbates come into play when lateral ordering and phase transformations are considered. 


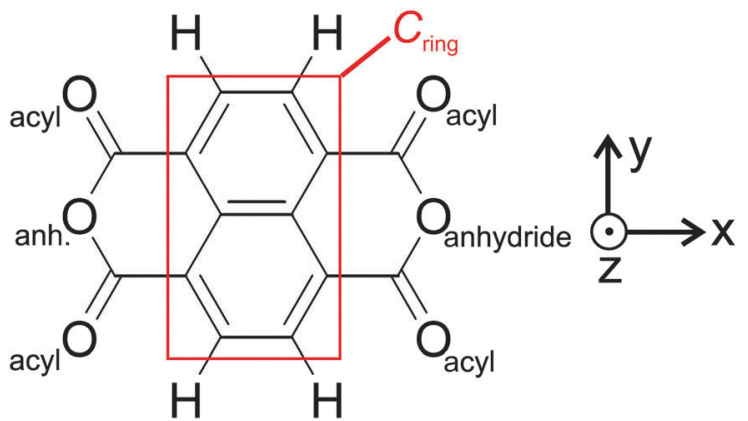

Scheme 1 1,4,5,8-naphthalene-tetracarboxylic dianhydride (NTCDA) with nomenclature. The 'long' axis $(x)$ connects the two anhydride oxygen atoms The short axis $(y)$ is aligned perpendicular to it. Coordinate system is chosen according to convention for surface science ( $z$-axis perpendicular to surface/ molecular plane of parallel oriented NTCDA).

As a model system for a moderately strong molecule-substrate coupling, we have chosen 1,4,5,8-naphthalene-tetracarboxylic dianhydride (NTCDA) molecules deposited onto a Ag(111) surface. $^{4-10}$ NTCDA is a planar molecule with $D_{2 \mathrm{~h}}$ symmetry (Scheme 1) thus exhibiting a short and a long molecular axis. NTCDA features two functionalities for bonding interactions with the surface: (i) The extended $\pi$-electron system associated with the naphthalene core and (ii) the acyl groups at the long ends of the molecule as part of the acid anhydride moieties. The suitability of NTCDA as a model system thereby stems from the possibility to employ these two functionalities, leading to a substrate-dependent delicate balance between covalent and dispersive bonding contributions. Modifications of the covalent bonds in the molecule, symmetry reduction upon adsorption, or localized bonding interactions with surface atoms may then be directly reflected in the vibrational spectrum and associated displacement patterns of normal modes. A detailed analysis of the IRAS spectra can therefore be used to test possible bonding configurations and obtain relevant information. In particular, changes in adsorption geometries could be identified as such, even in cases where the relative adsorbate-surface arrangement changes only marginally. These different adsorption geometries can have a crucial influence for the growth of the organic layer. Convenient features of IRAS thereby are its non-destructiveness and the ability to obtain information on mode polarizations and molecular symmetries by applying dipole and metal-surface selection rules.

We note that even though vibrational spectra inherently contain a multitude of specific information, especially related to the bonding configuration, an in-depth discussion usually requires comparison to computational modelling. The method of choice in the surface and materials sciences is density-functional theory (DFT). The main reason is the ability of DFT to treat realistic system sizes with good accuracy, ${ }^{11,12}$ especially for the case of organic molecules on metal surfaces, ${ }^{13}$ although major challenges for DFT have also been proposed recently. ${ }^{14}$ An important development in recent years was tackling the problem of missing description of dispersion interactions in most exchange-correlation functionals. Several groups work actively on solving this issue. ${ }^{15-19}$ Semi-empirical atomic pairwise approaches (e.g. DFT-D3) have demonstrated to work reliably for molecular as well as periodic systems in the past. ${ }^{20-26}$ Other methods are also in use for this methodological challenge. For PTCDA on different metal substrates, Ruiz et al. could show good agreement with experimental structures by an extended DFT-vdW approach. ${ }^{27}$ A recent study also found the DFT-D3 approach being accurate for this system. ${ }^{3}$

In such a combined approach, several challenges need to be addressed on both, the experimental and theoretical sides. Due to the large unit cells encountered in organic monolayer systems, the feasibility of accurate calculations represents a serious concern. This is aggravated by the large number of vibrational modes, which often cannot be attributed to localized functional groups but instead must be designated skeletal vibrations involving most atoms of the ring. ${ }^{28-30}$ The approach we are pursuing in this study is to develop a detailed understanding of the vibrational spectrum of the free molecule and - in a second step - analyse the changes in the spectrum upon adsorption on the metal surface. By combining the molecular with the periodic approach, we retrieve the best of both worlds, namely, the possibility to benchmark our DFT data in the molecular approach, while designating mode symmetry to all vibrations together with the consideration of the metallic environment in the periodic approach of the adsorbed molecule. This allows a quantification of the substrate's influence on the vibrational spectrum.

On the experimental side, the major difficulty concerns detection sensitivity, especially in conjunction with identifying weak modes with distinct polarizations being indicative of a possible reduction in adsorption site symmetry. In previous work we have demonstrated our capabilities to reproducibly prepare well defined organic monolayer films. ${ }^{31,32}$ Besides spot profile analysis low energy electron diffraction (SPA-LEED) and thermal desorption spectroscopy (TDS), IRAS has been established as a powerful technique to characterize organic monolayer films in terms of their vibrational properties. ${ }^{32}$

The system studied here has served as a model system before, in particular regarding structural and electronic properties. ${ }^{4-10}$ NTCDA on $\operatorname{Ag}(111)$ forms two long range ordered phases, a commensurate (relaxed) and an incommensurate (compressed) monolayer phase. ${ }^{4,5,31,32}$ Whereas the former is readily produced by annealing a thicker layer to $400 \mathrm{~K}$, the latter requires some more refined processing. ${ }^{31}$ In both cases a planar adsorption geometry prevails according to near edge X-ray absorption fine structure data ${ }^{6,8}$ Despite this parallel orientation, in-plane vibrational modes are found to display substantial dynamic dipole moments perpendicular to the surface, which is attributed to a pronounced NTCDA-Ag(111) interfacial dynamical charge transfer (IDCT) ${ }^{31}$ very much alike PTCDA/Ag(111) which displays a similar signature of vibrational bands. ${ }^{33-35}$ This process has been associated with a partially filled molecular orbital at the Fermi energy $\varepsilon_{\mathrm{F}}$ subject to variations in energetic position, depending on vibrational motion. ${ }^{36}$ Indeed, the NTCDA lowest unoccupied molecular orbital (LUMO) is found to shift down in energy upon adsorption and is located slightly below $\varepsilon_{\mathrm{F}},{ }^{7}$ based on ultraviolet photoemission spectroscopy (UPS). In our study this experimental finding is confirmed by DFT computations. ${ }^{36}$

From normal incidence X-ray standing wave (NIXSW) experiments, the vertical positions of carbon and oxygen atoms with 
respect to bulk $\mathrm{Ag}$ atom positions were determined. ${ }^{9,10}$ These represent key parameters to be compared with structural data derived from our calculations and will be discussed in-depth below.

In this study, we will present a detailed computational investigation of the structure and vibrational modes of free and adsorbed NTCDA molecules and compare these to the experimental IRAS measurements.

\section{Methods}

\section{Experimental details}

The IRAS experiments were carried out in a UHV chamber at a base pressure of $5 \times 10^{-11} \mathrm{mbar}$. The Fourier-transform infrared spectrometer (FTIR) was a Bruker IFS 66v/S with evacuable optics. The spectra were recorded using a liquid $\mathrm{N}_{2}$-cooled MercuryCadmium-Telluride detector (spectral range $600-5000 \mathrm{~cm}^{-1}$ ). We used polarized light throughout and selected a spectral resolution of $2 \mathrm{~cm}^{-1}$. IRAS measurements were obtained at a sample temperature of $28 \mathrm{~K}$. Temperature measurements used a K-type thermocouple welded to the edge of the $\mathrm{Ag}(111)$ sample. The $\operatorname{Ag}(111)$ crystal was cleaned by $\mathrm{Ar}^{+}$sputtering $\left(30 \mathrm{~min}\right.$ at $U_{\text {ion }}=$ $700 \mathrm{eV}, I_{\text {ion }}=3 \mu \mathrm{A}$, and $T_{\text {sample }}=373 \mathrm{~K}$ ) followed by annealing to $773 \mathrm{~K}$ for $5 \mathrm{~min}$. NTCDA was evaporated from a homemade thermal evaporator at $T_{\mathrm{NTCDA}}=390 \mathrm{~K}$ (deposition rate of $0.2 \mathrm{ML} \mathrm{min}^{-1}$ ) controlled by a Pt1000 temperature sensor. During evaporation, the background pressure typically increases by $\Delta p=1 \times 10^{-10}$ mbar.

\section{Computational details}

(a) Non-periodic calculations. Geometry optimizations using the known point-group symmetry of free NTCDA $\left(D_{2 \mathrm{~h}}\right)$ have been carried out using the Gaussian09 optimizer (standard convergence criteria) ${ }^{37}$ with Turbomole ${ }^{38}$ (version 6.4) energies (convergence criterion $10^{-8}$ a.u.) and gradients with the functional and basis set combination $\mathrm{PBE}^{39} / \mathrm{def}_{2}-\mathrm{TZVPP}^{40}$ considering dispersion effects by applying the DFT-D3 method $^{41}$ with an improved damping function ${ }^{42}$ for the sake of consistency with the periodic calculations. This level of approximation is denoted by PBE-D3/TZ in the following. Further calculations have been carried out with the MP2 method as implemented in Gaussian09 with the same basis set (MP2/TZ). Stationary points were characterized as minima by calculating the Hessian matrix analytically, additionally providing the vibrational spectrum. ${ }^{43}$ The resolution of the identity method has been applied for the PBE calculations to approximate fourcenter integrals. $^{44}$

(b) Periodic calculations. The interaction of NTCDA with the $\mathrm{Ag}(111)$ surface was studied by means of DFT calculations employing the Vienna Ab Initio Simulation Package (VASP 5.2), ${ }^{45}$ which uses periodic boundary conditions (indicated by subscript "PBC" in this work). The generalized gradient approximation (GGA) using the exchange-correlation functional proposed by Perdew, Burke and Ernzerhof (PBE) ${ }^{39}$ was used in combination with a plane-wave basis set. The projector-augmented wave (PAW) method ${ }^{46,47}$ was applied and enabled a truncation of the plane-wave basis set at a kinetic energy of $350 \mathrm{eV}$. This value was found to be sufficiently accurate for metal-organic interfaces in the past. $^{48-50}$

The projection operators for the non-local part of the pseudopotentials were evaluated in real space with the exception of the linear response calculations. The Brillouin zone for the adsorption studies was sampled by a $\Gamma$-centred $3 \times 3 \times 1 k$-point mesh generated via the Monkhorst-Pack method. ${ }^{51}$ The Ag bulk structure was optimized with $E_{\text {cutoff }}=350 \mathrm{eV}$ and a $\Gamma$-centred $25 \times 25 \times 25 k$-mesh. All calculations employed the precision parameter setting "accurate".

Structural optimization was performed starting from different initial structures by means of a conjugate-gradient or quasi-Newton algorithm for ionic positions and cell parameters until the forces converged to $1 \times 10^{-2} \mathrm{eV} \AA^{-1}$ and the energy during SCF cycles converged to $10^{-5} \mathrm{eV}$. Vibrational frequencies at the $\Gamma$-point of the periodic cell were calculated through building up the force constant matrix either via a density-functional perturbation theory (DFPT) ${ }^{52-54}$ and alternatively via a finite difference approach. IR intensities were calculated in a dynamic dipole-derivative ansatz considering only the component of the dipole moment change normal to the surface plane upon displacement. The vibrational frequencies have been determined from a partial hessian obtained by displacing only the NTCDA-atoms. A test with inclusion of the top-most Ag-layer in the force-constant calculation did not show a significant change of the frequencies $\left(\Delta \nu=-0.05 \mathrm{~cm}^{-1}\right)$.

The adsorption energies were calculated by subtracting the energy of the surface-adsorbate complex from the total energies of the relaxed $\mathrm{Ag}(111)$ substrate layers and the relaxed adsorbate molecule. Dispersion effects have been considered by the semi-empirical correction scheme proposed by Grimme in the D3 formulation ${ }^{41}$ (with "Becke-Johnson"-type damping function $)^{42}$ in an implementation by Möllmann and Grimme. ${ }^{23}$ This methodology is abbreviated PBE-D3 ${ }_{\mathrm{PBC}}$ in the following. For comparison, some values were derived with the older DFT-D2 version as well $\left(\mathrm{PBE}-\mathrm{D} 2_{\mathrm{PBC}}\right){ }^{55}$ All vibrational calculations were carried out in the harmonic approximation and representations of IR-spectra used a Lorentzian broadening with a $5 \mathrm{~cm}^{-1}$ full width at half maximum (FWHM). The intensities of IR-active modes are normalized to the most intense mode.

AIM atomic charges have been computed with a grid-based algorithm. ${ }^{56}$ The density of states (DOS) was obtained with the lobster 2.0 .0 code $^{57-59}$ by projection to a local basis set. ${ }^{60}$

(c) Validation of computational methods - Ag bulk and Ag(111) surface. Initially, the silver bulk phase was optimized starting from the experimental lattice constant. Results were derived with (D2, D3) and without dispersion correction scheme to the PBE energies (Table S1, ESI $\dagger$ ). As it turns out, the inclusion of dispersion corrections improves the agreement with experimental parameters (lattice constant and bulk modulus) although dispersion forces are not expected to make large contributions to bulk metals. While the (old) D2 correction scheme provides fixed $\mathrm{C}_{6}$-parameters, the more recent $\mathrm{D} 3$ scheme considers the environment of the atoms in the calculation of the dispersion energy. As the improvement toward experimental parameters suggests, this seems to allow the description of what has been termed "screening" in studies with other dispersion correction 
schemes at least in a local environment. ${ }^{61}$ Thus, in the following we used the lattice parameters optimized with the PBE-D3 scheme for the surface adsorption part of the study.

Regarding the $\mathrm{Ag}(111)$ surface, relaxation effects are to be expected relative to the bulk positions. The four-layer slab model employed here (two bottom layers fixed) gives the correct relaxation sign and magnitude for the change of the first $\left(\Delta d_{12} / d_{\mathrm{b}}=-0.9 \%\right)$ and the second interlayer spacing $\left(\Delta d_{23} / d_{\mathrm{b}}=\right.$ $-0.3 \%)$ relative to the bulk spacing $\left(d_{\mathrm{b}}\right)$, when compared to LEED $^{62}$ and X-ray scattering studies. ${ }^{63}$ Note that the LEED study showed that the third interlayer spacing is the same as the bulk interlayer spacing thus legitimating our four-layer slab approach. More refined results would need the consideration of finitetemperature effects which have been discussed in the past but are not the aim of this study. ${ }^{64}$

\section{Results and discussion}

\section{Computational results}

NTCDA is known to adsorb reversibly onto $\mathrm{Ag}(111)$ with desorption temperatures of about $430 \mathrm{~K}$ (low-coverage limit). The interaction of the molecules with the substrate thereby comprises two contributions, (i) the ubiquitous van der Waals interaction and (ii) a covalent binding component stemming from the interaction of the acyl groups at both ends of the molecule with Ag substrate atoms. The strength of the latter interaction is underlined by a pronounced out-of-plane displacement of the acyl oxygen atoms towards the surface, which is accompanied by an opposite vertical shift of the anhydride oxygen atoms. ${ }^{9,10}$ Specifically, the NIXSW experiments found an overall distance of $2.997 \AA$ for the naphthalene core, a value much smaller than expected for van der Waals interactions alone. These observations are very similar to the findings of PTCDA, the perylene equivalent of NTCDA. ${ }^{65,66}$ We stress that the NIXSW measurements used an averaged $\mathrm{C}_{1 \mathrm{~s}}$ signal for all carbon atoms of NTCDA, therefore comprising not only the naphthalene core but also the anhydride carbon atoms. ${ }^{67}$ Therefore, we will discuss values for the naphthalene core $\left(\mathrm{C}_{\text {Ring }}\right.$ in Scheme 1) as well as averaged values for all carbon atoms $\left(\mathrm{C}_{\mathrm{all}}\right)$.

In IR spectra, the donor-acceptor bonds between $\mathrm{Ag}$ and the acyl groups are reflected in a bold red shift of $\nu_{\mathrm{C}=\mathrm{O}}$ frequencies from the gas phase values, as proclaimed in a very recent study of ours. ${ }^{31}$ In the present study, a theoretical analysis of NTCDA structure and vibrations, both of the free molecule and adsorbed on $\mathrm{Ag}(111)$ will be presented. The arrangements of NTCDA on Ag(111) which have been investigated computationally are displayed in Fig. 1. The chosen setup has two advantages: (i) there will be only one type of adsorption site for NTCDA (as opposed to the relaxed NTCDA/Ag(111) monolayer with two inequivalent sites), which reduces the calculation

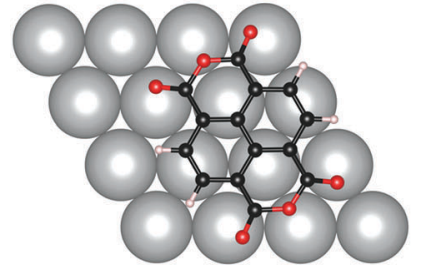

a) bridge $_{1}$ $E_{\text {ads }}=202 \mathrm{~kJ} \mathrm{~mol}^{-1}$

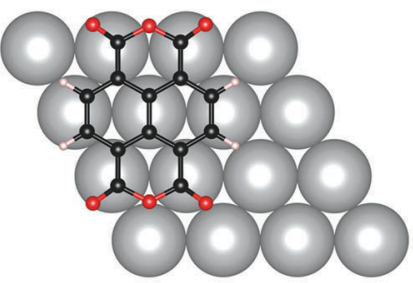

d) top $_{2}$

$E_{\mathrm{ads}}=170 \mathrm{~kJ} \mathrm{~mol}^{-1}$

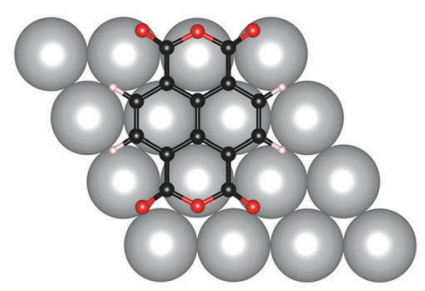

b) bridge 2 $E_{\text {ads }}=168 \mathrm{~kJ} \mathrm{~mol}^{-1}$

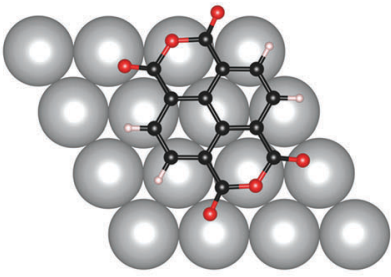

c) top 1 $E_{\text {ads }}=196 \mathrm{~kJ} \mathrm{~mol}^{-1}$

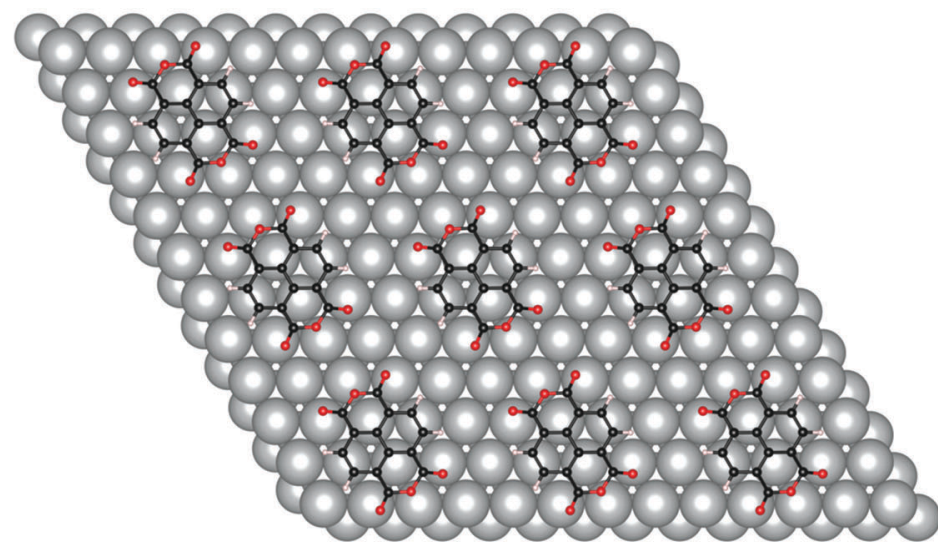

e) $(3 \times 3)$ supercell for bridge $_{1}$-sites

Fig. 1 Adsorption sites of NTCDA on Ag(111), together with adsorption energies in $\mathrm{kJ} \mathrm{mol}^{-1}\left(\mathrm{a}-\mathrm{d}\right.$ ), and supercell for bridge ${ }_{1}$-site adsorption of NTCDA (e), visualising the surface arrangement as calculated using DFT (PBE-D3 $3_{\mathrm{PBC}}$ ). Nomenclature analogous to Alkauskas et al. ${ }^{68}$ referring to the position of the $\mathrm{C}-\mathrm{C}$ bond relative to the topmost Ag-layer. Bridge ${ }_{1}$ and top $\mathrm{p}_{1}$ adsorption geometries have the molecular axis parallel to the Ag rows, while for bridge 2 and top $_{2}$ configurations the axis is perpendicular to Ag rows. 
effort substantially and simplifies the derived vibrational spectra. (ii) Lateral interactions between neighbouring NTCDA molecules, especially $\mathrm{H}$-bonding, can be neglected. In the experiment, these conditions have been met by sub-monolayer deposition of NTCDA onto $\mathrm{Ag}(111)$ at low temperature $(T \approx 28 \mathrm{~K})$. In the following, we will first discuss the structure and molecular distortion of adsorbed NTCDA and then present our vibrational mode analysis. Based on calculations of single (gas phase) NTCDA molecules, the effect of bonding to $\mathrm{Ag}(111)$ is derived.

(a) Structure of free NTCDA and NTCDA adsorbed on $\mathbf{A g}(\mathbf{1 1 1})$. In a first step, the free NTCDA molecule was optimized with non-periodic $a b$ initio methods with results summarized in Fig. 2. DFT calculations (PBE-D3/TZ) are compared to wave function-based results (MP2/TZ). While PBE-D3/TZ leads to slightly longer bonds, the overall agreement is very good and deviations are below $0.01 \AA$. The derived values also compare well to results from an X-ray diffraction study of the molecular crystal by Born and Heywang ${ }^{69}$ included in Fig. 2. This comparison in particular confirms that both methods are suitable for reproducing the NTCDA molecular structure with high accuracy.

The next step in the computations comprises the investigation of NTCDA adsorption on the Ag(111) surface. In the course of the structural optimization, the NTCDA molecular structure and the coordination with respect to $\mathrm{Ag}(111)$ was allowed to relax. Our use of dispersion corrected DFT ensures a constraints-free structural optimization to reach the energetic minimum. In order to account for the elasticity of the $\mathrm{Ag}(111)$ surface, the upper two layers were allowed to relax as well, while the two bottom layers have been kept at (calculated) bulk positions. For further discussion of the bulk and surface computations, see the computational details section.

Several possible adsorption sites were tested in the calculations as shown in Fig. 1. Adsorption energies (PBE-D3 ${ }_{\mathrm{PBC}}$ ) are also included in Fig. 1 and will be discussed below. It can be deduced from these data that an adsorption geometry with the central $\mathrm{C}=\mathrm{C}$ bond located above a bridge site of $\mathrm{Ag}(111)$ and with the (long) $\mathrm{O}-\mathrm{O}$ axis aligned along $\mathrm{Ag}$ atom rows is most stable $\left(E_{\mathrm{ads}}=202 \mathrm{~kJ} \mathrm{~mol}^{-1}\right.$, Fig. 1a, bridge $\left.{ }_{1}\right)$. We identified an alternative adsorption geometry

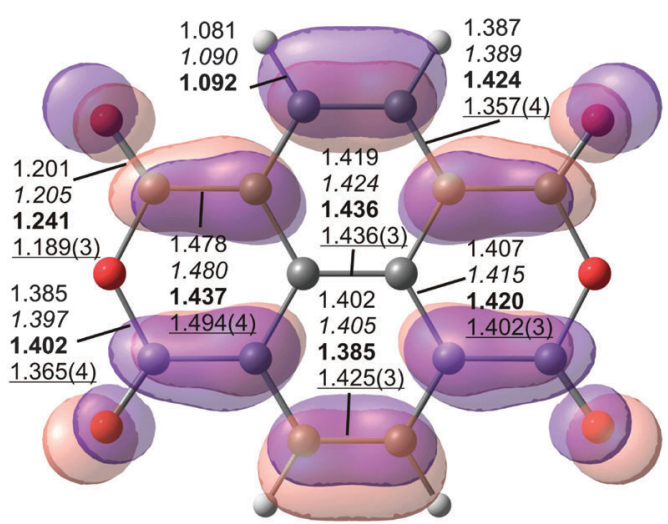

Fig. 2 Structural parameters of NTCDA (top view) with the LUMO overlaid (isosurface with contour value 0.03 ). Calculated bond lengths of the free molecule (MP2/TZ and PBE-D3/TZ in italics, $D_{2 h}$ ) and the adsorbed molecule (PBE-D3 ${ }_{P B C}$ in bold, $C_{2 v}$ ) are given together with data from $X$-ray diffraction ${ }^{69}$ (underlined) of the NTCDA molecular crystal $\left(C_{i}\right)$. with the central $\mathrm{C}-\mathrm{C}$ bond located above an $\mathrm{Ag}$ atom and with the $\mathrm{O}-\mathrm{O}$ axis again aligned along $\mathrm{Ag}$ atom rows (Fig. $1 \mathrm{c}$, top ${ }_{1}$ ) showing a marginally lower adsorption energy $\left(E_{\mathrm{ads}}=196 \mathrm{~kJ} \mathrm{~mol}^{-1}\right)$. Substantially weaker bonding, on the other hand, is found for both of these two sites but with a $90^{\circ}$ rotated azimuthal orientation $\left(E_{\text {ads }}=168 \mathrm{~kJ} \mathrm{~mol}^{-1}\right.$ (bridge 2$), 170 \mathrm{~kJ} \mathrm{~mol}^{-1}\left(\right.$ top $\left.\left._{2}\right)\right)$.

Upon adsorption of the NTCDA molecule on the $\operatorname{Ag}(111)$ surface, the bonds within the molecule are distorted as depicted in Fig. 2 for the most stable adsorption geometry. In order to explain the distortion pattern it is instructional to recall the (partial) filling of the LUMO of the free molecule upon surface adsorption. This experimental observation ${ }^{7,70}$ is confirmed by our calculations (see below). Therefore, we have overlaid the isosurface plot of the LUMO of NTCDA with the molecular structure (Fig. 2). The filling of the molecular LUMO orbital upon adsorption leads to an increase in bond strength (and thereby a shortening) of those bonds where additional electron density is accumulated. Accordingly, all bonds at nodes of this orbital become elongated with respect to the free molecule. The comparison of $\mathrm{PBE}-\mathrm{D} 3_{\mathrm{PBC}}$ values for the free (computed with PBC in a large box) and adsorbed molecule in Fig. 2 fully supports this hypothesis.

In addition to the in-plane distortion, the NTCDA molecule significantly bends towards the surface (Fig. 3). This reduces the (local) molecular symmetry from $D_{2 \mathrm{~h}}$ to (approximately) $C_{2 \mathrm{v}}$. Minor deviations from the exact $C_{2 v}$ symmetry are for example differences of the vertical distances for the $\mathrm{C}-\mathrm{H}$ groups from $\mathrm{Ag}$ surface atoms amounting to $\Delta z \approx 0.002 \AA$, i.e. the variation is less than $0.1 \%$. This could be due to the inequivalent threefold hollow sites below the two carbon rings of the naphthalene core (Fig. 3b) which would reduce the molecular symmetry to $C_{\mathrm{S}}$. The side view (Fig. 3c) clearly describes the downward bending of the acyl groups. The distortions in $z$-direction are reproduced in more detail in Fig. 3a and b. While the carbon and oxygen atoms of the acyl groups are significantly bent toward the surface, the anhydridic oxygen atoms experience an opposite bending with respect to the neighbouring C-atoms. Due to an overall warping of the molecular plane, these are nonetheless located below the plane, defined by the average height of carbon atoms forming the naphthalene core $\left(\mathrm{C}_{\mathrm{Ring}}\right)$. This is in accordance with previous experimental and theoretical studies related to NTCDA and PTCDA monolayers. ${ }^{3,9,10,48,65,66,71}$ Moreover, the carbon skeleton displays strong distortions as well. Besides the downward bending of the acyl group it is in particular the inner $\mathrm{C}=\mathrm{C}$ bond being bent upward relative to the above-defined molecular plane (atom positions 4 and 5 in Fig. 3a).

The surface itself also warrants attention. In Fig. 4 we have visualized the local vertical relaxations of the $\mathrm{Ag}(111)$ surface covered by NTCDA in a similar way as Bauer et al. did for PTCDA on $\operatorname{Ag}(111) .{ }^{3}$ In the course of the adsorption process, the $\mathrm{Ag}(111)$ surface is found to display considerable buckling, indicative of directional adsorbate-substrate bonds beyond pure van der Waals interactions. Specifically, the covalent bonds of acyl oxygen atoms cause a protrusion of nearby $\mathrm{Ag}$ atoms which is nevertheless surprisingly small $(\Delta h \approx 0.005 \AA)$. 

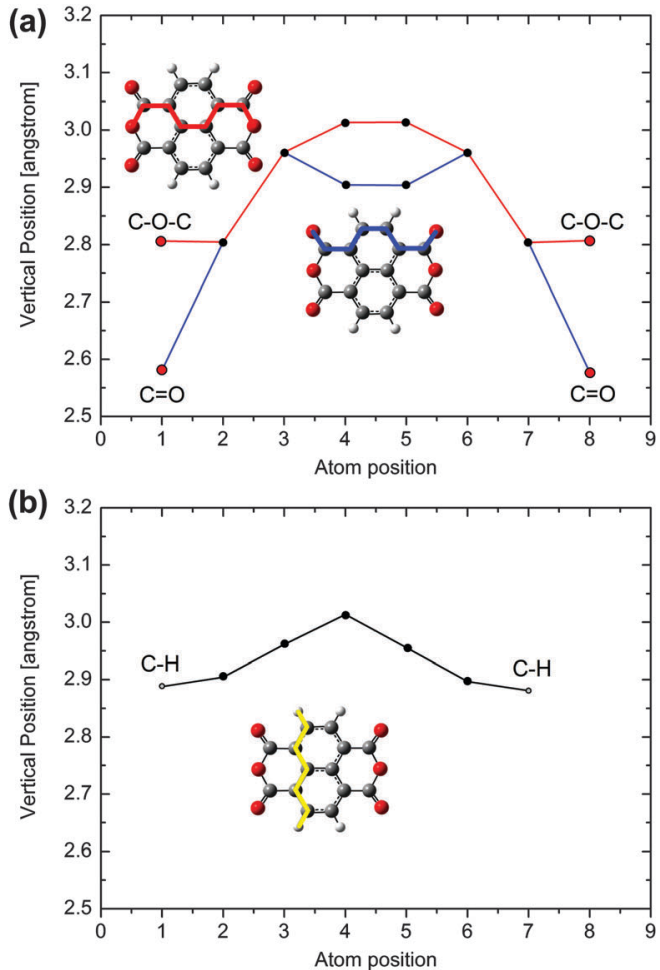

(c)

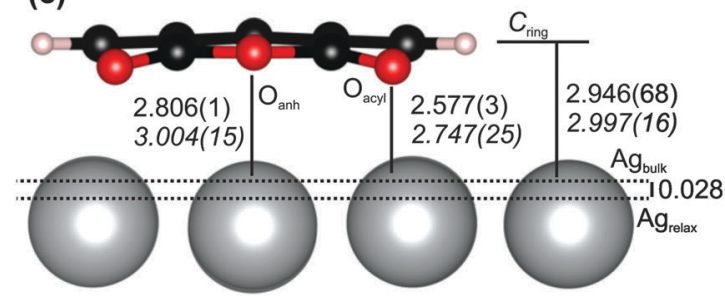

Fig. 3 Calculated (PBE-D3 ${ }_{\mathrm{PBC}}$ ) vertical distances of the various atoms of adsorbed NTCDA/Ag(111) in bridge ${ }_{1}$ mode. Shown are cuts through the molecule (a) connecting the $\mathrm{O}_{\text {acyl }}$ and $\mathrm{O}_{\text {anh }}$ atoms, and (b) connecting the hydrogen atoms together with (c) the most important vertical computed distances compared to NIXSW experiments. ${ }^{10}$ All vertical distance values refer to the bulk-extrapolated $\mathrm{Ag}$ surface atom positions.

$\mathrm{Ag}$ atoms below the central $\mathrm{C}-\mathrm{C}$ backbone, on the other hand, experience a significant repulsion, pushing those $\mathrm{Ag}$ atoms below the average Ag surface plane $(\Delta h \approx-0.074 \AA)$. The overall corrugation of the $\mathrm{Ag}$ surface is included in Table 1: we find a pronounced buckling of $0.134 \AA$ for the topmost layer and $0.025 \AA$ for the second layer for NTCDA occupying the bridge $_{1}$ adsorption site; for the top ${ }_{1}$ site these numbers transform to $0.208 \AA$ and $0.076 \AA$, respectively.

In accordance with similar observation for PTCDA on $\operatorname{Ag}(111)^{3}$ we conclude that the attraction of the molecule towards the surface by the acyl oxygen atoms leads to increased Pauli repulsion between the $\pi$-electrons of the naphthalene core and the $\mathrm{Ag}$ atoms underneath. Interestingly, $\mathrm{Ag}$ atoms below the $\mathrm{HC}=\mathrm{CH}$ subunits display a particularly severe up-shift. This is most probably an effect to counterbalance the downward motion of the $\mathrm{Ag}$ atoms underneath the molecule's centre. The surface corrugation due to electronic effects in the molecule-substrate

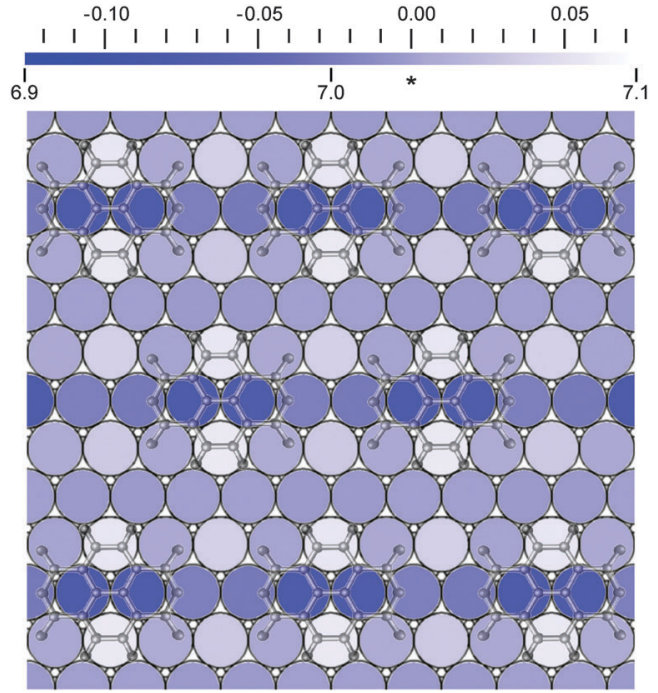

Fig. 4 Corrugation of the $A g(111)$ surface stemming from PBE-D3 ${ }_{P B C}$ computations with vertical positions of $\mathrm{Ag}$ atoms displayed in different shades of blue according to the colour bar. The numbers underneath of the colour scale bar refer to the distance in $\AA$ from the bottom layer of our four-layer Ag slab. The average vertical position of the Ag(111) surface layer $(7.026 \AA)$ is denoted by an asterisk (*). This value is used as a reference for the upper scale. The arrangement of the NTCDA molecules is overlaid in light grey. The layer spacing of the Ag bulk in our calculation was $\Delta d_{111}=$ $2.352 \AA$, which compares favourably with the experimental value of $2.349 \AA$.

Table 1 Compilation of calculated (PBE-D3 ${ }_{\mathrm{PBC}}$ ) and experimental (NIXSW) vertical distances $d(A-B)$ for NTCDA on the Ag(111) surface. The choice of reference layer $\left(z_{\text {Ref }}=0\right)$ enables direct comparison to experiment (Ag bulk in Fig. 3). All values are given in $\AA$

\begin{tabular}{|c|c|c|c|}
\hline \multirow[b]{2}{*}{$\mathrm{A}-\mathrm{B}$} & \multicolumn{2}{|c|}{ PBE-D3 $_{\mathrm{PBC}}$, dilute layer ${ }^{a}$} & \multirow{2}{*}{$\begin{array}{l}\text { Experiment, } \\
\text { dense layer }^{10}\end{array}$} \\
\hline & Bridge $_{1}$ site & Top $_{1}$ site & \\
\hline $\mathrm{O}_{\mathrm{acyl}}-\mathrm{Ag}$ & $2.577 \pm 0.004$ & $2.622 \pm 0.003$ & $2.747(25)$ \\
\hline $\mathrm{O}_{\mathrm{anh}}-\mathrm{Ag}$ & $2.806 \pm 0.001$ & $2.798 \pm 0.001$ & $3.004(15)$ \\
\hline $\mathrm{C}_{\mathrm{all}}-\mathrm{Ag}^{b}$ & $2.905 \pm 0.145$ & $2.890 \pm 0.090$ & $2.997(16)$ \\
\hline $\mathrm{C}_{\text {ring }}-\mathrm{Ag}^{c}$ & $2.946 \pm 0.068$ & $2.925 \pm 0.051$ & \\
\hline $\mathrm{O}_{\text {acyl }}-\mathrm{C}_{\text {ring. }}$ & $-0.369 \pm 0.070$ & $-0.307 \pm 0.052$ & $-0.25(3)$ \\
\hline $\mathrm{O}_{\text {anh }}-\mathrm{C}_{\text {ring }}$ & $-0.140 \pm 0.068$ & $-0.134 \pm 0.051$ & $\approx 0^{d}$ \\
\hline $\mathrm{O}_{\mathrm{anh}}-\mathrm{O}_{\text {acyl }}$ & $0.230 \pm 0.004$ & $0.173 \pm 0.005$ & $0.26(4)$ \\
\hline$\delta z_{\mathrm{Ag}-\mathrm{Ag}}^{e}$ & 0.134 & 0.208 & \\
\hline$\delta z_{\mathrm{Ag} / \mathrm{sub}-\mathrm{Ag} / \mathrm{sub}}{ }^{f}$ & 0.025 & 0.076 & \\
\hline
\end{tabular}

${ }^{a}$ Given are averaged values for equivalent atoms and the maximum deviation from this average. ${ }^{b}$ Position of all carbon atoms averaged. ${ }^{c}$ Position of ring carbon atoms averaged $\left(\mathrm{C}_{\mathrm{ring}}\right)$ - see Scheme $1 .{ }^{d}$ The difference is smaller than the uncertainty in the measurements. ${ }^{e}$ Difference in $z$-coordinates of $\mathrm{Ag}$ atoms (corrugation) of the topmost layer. ${ }^{f}$ Difference in $z$-coordinates of $\mathrm{Ag}$ atoms (corrugation) of the subsurface layer.

interaction is quite pronounced and deserves further analysis for similar systems in the future.

The derived structural data for the most stable adsorption configuration (bridge ${ }_{1}$ ) allows a direct comparison to NIXSW measurements obtained for $\operatorname{NTCDA} / \operatorname{Ag}(111) \cdot{ }^{9,10}$ We note beforehand that the NIXSW data have been obtained from dense layers with H-bonding among neighbouring NTCDA molecules. The competition of such interactions with covalent 
bonding of $\mathrm{O}_{\text {acyl }}$ to $\mathrm{Ag}$ should weaken the $\mathrm{Ag}-\mathrm{O}_{\text {acyl }}$ bond and most likely lead to a reduced downward bending of the acyl-oxygens. This hypothesis is based on NIXSW results by Kilian et al. ${ }^{71}$ referring to PTCDA/Ag(111).

Both, experimental and the (averaged) computational adsorbatesurface distances are compiled in Table 1 (for data on all adsorption geometries investigated see Table S2 in the ESI $\dagger$ ). Since the NIXSW experiment did not distinguish between the various inequivalent $\mathrm{C}$-atoms we introduced the values $\mathrm{C}_{\text {all }}$ and $\mathrm{C}_{\text {Ring }}$ to quantify the average vertical distances of carbon entities defined in Scheme 1. In order to allow a proper comparison to experimental NIXSW data we have introduced a hypothetical Ag surface plane Ag* which corresponds to the extrapolated positions of $\mathrm{Ag}$ bulk atoms. The difference with respect to the true averaged $\mathrm{Ag}$ surface layer $(\Delta h=0.028 \AA)$ corresponds to the $\mathrm{Ag}(111)$ surface relaxation (surface interlayer distance shorter than bulk value).

First of all, the PBE-D3 values provided in this study agree formidably with the experimental values. Note that a structural optimization without dispersion correction (PBE) gives unreasonably long surface-adsorbate distances, $d\left(\mathrm{C}_{\text {Ring }}-\mathrm{Ag}\right)=3.534 \AA$ (further details in Table S2 in the ESI $\dagger$ ). Thus, we confirm the finding that pure GGA functionals are not able to describe weak adsorbate-surface complexes correctly. ${ }^{72}$ The computed distance for the best binding site (bridge ${ }_{1}$ ) $\mathrm{C}_{\text {all }}=2.905 \AA$ for NTCDA/Ag(111) using PBE-D3 ${ }_{\mathrm{PBC}}$ is in accord with the experimentally found value of $2.997 \AA$. Both, experiment and theory confirm that the acyl oxygens are located substantially below the carbon plane as outlined above. The computed averaged $\mathrm{Ag}-\mathrm{O}_{\text {acyl }}$ distance is $2.577(3) \AA$ while the $\mathrm{Ag}-\mathrm{O}_{\text {anh }}$ distance is

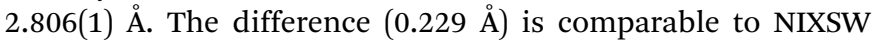
results $(0.257 \AA)$. Similar values are found for the energetically close-lying top ${ }_{1}$ adsorption geometry. The $\mathrm{Ag}-\mathrm{O}_{\text {acyl }}$ distance is thereby at the upper end of the range for typical distances in molecular complexes of silver 2.4-2.6 $\AA^{73}$ No reference data are available for $\mathrm{Ag}-\mathrm{O}_{\mathrm{anh}}$-bonds in the solid state.

Actually, the calculations predict a notably stronger downward bending of the oxygen atoms as compared to the experiment. In addition, the anhydride oxygen atoms are located distinctly below the carbon plane while they are found above the carbon plane in the NIXSW data. These discrepancies are attributed to a severe overall bending of the entire NTCDA molecule. As our calculated numbers refer to NTCDA separated by at least one $\mathrm{Ag}$ atom row, they correspond to layers with negligible direct lateral interactions; experimental NIXSW data, on the other hand, were obtained from dense NTCDA-layers with substantial H-bonding between neighbouring molecules. 9,10

According to our findings, the warping of the molecular plane is substantially stronger for the less densely packed arrangement of NTCDA molecules in our calculation. Unfortunately, low-coverage NIXSW data (isolated NTCDA adsorbates) which would compare directly with our theoretical values are not available. We therefore also investigated the relaxed monolayer phase of NTCDA on $\mathrm{Ag}(111)$ with distances between oxygen and hydrogen atoms of neighbouring NTCDA molecules amounting to 2-2.5 $\AA$, i.e. with H-bonding contributions. This corresponds to the most recently found structure for the relaxed monolayer. ${ }^{32}$ For such dense layers the overall distance to the $\mathrm{Ag}(111)$ surface increases by $0.05-0.10 \AA$, in line with a weaker Ag-NTCDA interaction in this complex. Moreover, the warping of the molecular plane and the downward bending of the acyl oxygens is reduced notably.

Additional confirmation for our hypothesis of a more severe bending of adsorbed NTCDA in the absence of H-bonding (isolated adsorbed molecules) comes from previous studies on PTCDA/Ag(111) data. ${ }^{65,66,71}$ Specifically, two NIXSW data sets comprising dense as well as dilute layers exist and they have been used to estimate trends related to the effect of intermolecular interactions within well-ordered layers, as compared to less dense, disordered agglomerates of adsorbed molecules. These findings suggest that a dilute species is interacting more strongly with $\mathrm{Ag}(111)$ and experiences more severe distortions. When comparing our calculated structure data to NIXSW results (obtained for a dense relaxed NTCDA monolayer on $\operatorname{Ag}(111)$ ) the influence of lateral interactions should therefore be kept in mind.

According to Stadler et al., the NIXSW data of NTCDA on $\mathrm{Ag}(111)$ show a rather small coherent fraction for the $\mathrm{C}_{1 \mathrm{~s}}$ signal, indicative of a relatively large spread in vertical distances of the individual C-atoms. ${ }^{10}$ This finding is in perfect agreement with the substantial warping of the NTCDA carbon skeleton according to our calculations. Recently, such a warping has also been suggested for PTCDA on various Ag surfaces based on dispersion-corrected DFT calculations. ${ }^{3}$

The adsorption energies for the arrangements studied in this work (see Fig. 1) are derived from PBE-D3 ${ }_{\mathrm{PBC}}$ structural optimizations. Positive energies thereby represent stable surface-adsorbate complexes. The most favourable adsorption site is the bridge ${ }_{1}$ site aligned with the long axis along $\mathrm{Ag}$ atom rows (adsorption energy equals $202 \mathrm{~kJ} \mathrm{~mol}^{-1}$ ). Only slightly less stable is the equally aligned top ${ }_{1}$ site $\left(196 \mathrm{~kJ} \mathrm{~mol}^{-1}\right)$ while the other two sites investigated (bridge $\mathrm{b}_{2}$ and top $\mathrm{p}_{2}$, both aligned perpendicular to $\mathrm{Ag}$ atoms rows) are far less stable. Thus the computational data suggest that NTCDA adsorbs either on a bridge $_{1}$ or a top ${ }_{1}$-site. In a test calculation without dispersion correction (PBE), the adsorption energy even turns negative with $-2.7 \mathrm{~kJ} \mathrm{~mol}^{-1}$, in accordance with the unreasonably large adsorbate-surface distance $\mathrm{C}_{\mathrm{Ring}} \mathrm{-Ag}=3.534 \AA$ (see above) and similar findings for PTCDA/Ag(111) using a comparable theoretical approach. ${ }^{74}$

A closer look at the relative positions of the $\mathrm{O}_{\text {acyl }}$ atoms and the topmost layer of the silver slab reveals that in these two geometries, all four doubly-bonded oxygen atoms are located on top of $\mathrm{Ag}$ atoms, while they are displaced in the other two arrangements. This is another indication that the $\mathrm{Ag}-\mathrm{O}_{\mathrm{acyl}}$ interaction is decisive for the site preference of adsorbed NTCDA. Experimentally, an estimate of the adsorption energy is available from temperature programmed desorption (TPD) investigations of a dilute monolayer giving a value of $112 \pm 10 \mathrm{~kJ} \mathrm{~mol}^{-1}$ $(1.14 \mathrm{eV}) .{ }^{4}$ This value reported by Stahl et al., however, appears much too low. In our opinion, the very gradual initial slope in the measurements presented points toward a heterogeneous surface layer or a mixture of different phases. As a consequence of 
the shallow initial slope of the desorption signal an unusually low prefactor of about $10^{12} \mathrm{~s}^{-1}$ is deduced. As our submonolayer TD spectra show this long tail towards the low temperature side as well, we decided to take the well-shaped TD spectra of the likewise parallel oriented NTCDA bilayer to obtain a lower bound for the adsorption energy of the NTCDA/Ag(111) monolayer species. Analysing the initial slope of the bilayer desorption signal yields an adsorption energy of $121 \pm 10 \mathrm{~kJ} \mathrm{~mol}^{-1}$ (1.26 eV per molecule) and a prefactor of $1.5 \times 10^{17} \mathrm{~s}^{-1}$ (such high prefactors are quite common for large molecules desorbing from metal surfaces ${ }^{7-77}$ ). According to the higher desorption temperatures of monolayer NTCDA and assuming a similar prefactor, we estimate the adsorption energy of monolayer NTCDA/Ag(111) to equal about $150 \mathrm{~kJ} \mathrm{~mol}^{-1}(1.56 \mathrm{eV}$ per molecule) which is much closer to the calculated values.

It was already discussed in previous publications regarding NTCDA and PTCDA on various metal substrate surfaces that the LUMO of the free molecule may become partially occupied upon adsorption. ${ }^{3,7,48,70,71}$ In our study such behaviour is clearly confirmed as indicated by the partially occupied band (occupation $1.42 \mathrm{e}$, band located $0.04 \mathrm{eV}$ below $E_{\text {Fermi }}$ ) shown in Fig. 5 exhibiting mainly LUMO (NTCDA) - character. This can also be seen in the partial density of states (pDOS) shown in Fig. 5c, which exhibits significant NTCDA-character of the states around the Fermi level $\left(E_{\mathrm{Fermi}}\right)$.

To quantify this partial filling of the LUMO and the net charge transfer between surface and adsorbate, we derived partial charges in the framework of the atoms-in-molecules (AIM) theory. The AIM results suggest a negatively charged NTCDA molecule with a charge of $\Delta q=-0.94 e$. This can be compared to the estimate of $-0.35 e$ by charge summation ${ }^{78}$ and the value of $-1.00 e$ for PTCDA on $\mathrm{Ag}(111)$ derived by a similar AIM approach as followed here. ${ }^{3}$ It can be concluded

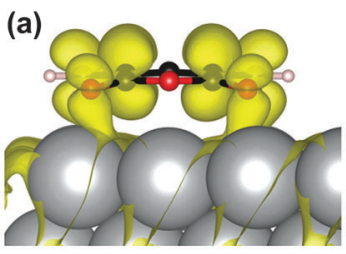

(b)

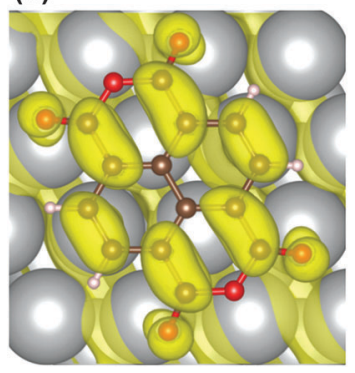

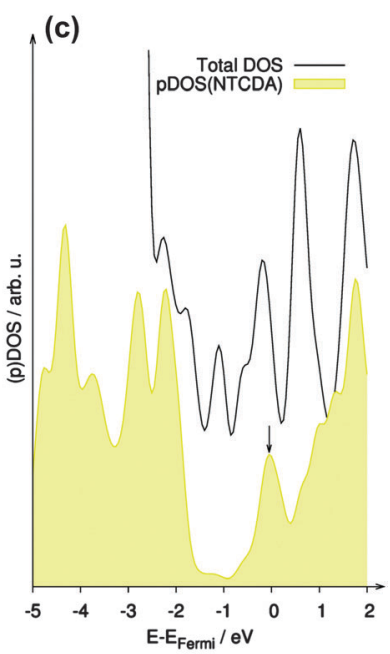

Fig. 5 Band at the $\Gamma$-point mainly consisting of the former LUMO $(0.04 \mathrm{eV}$ below $E_{\text {Fermi, }}$ indicated by black arrow in DOS plot) of NTCDA on $\mathrm{Ag}(111)$, which becomes partially occupied (1.42 e) upon adsorption on $\mathrm{Ag}(111)$ in side (a) and top (b) view together with (c) total density of states (DOS) of the complete system (black line) and partial DOS of the adsorbate (yellow). that NTCDA shows a similar charge transfer compared to PTCDA and thus differs considerably from a purely physisorbed state.

The accurate reproduction of experimental structural data of NTCDA adsorbed on the $\mathrm{Ag}(111)$ surface underlines the importance and accuracy of dispersion correction methods in DFT-based calculations to arrive at a trustworthy description of adsorbed large organic molecules. In addition to the NIXSW experiments, ${ }^{10}$ the calculations can then help in identifying subtle details of the adsorption geometry which are not (yet) resolved in the experimental studies, such as the corrugation of the Ag surface or of the carbon skeleton of adsorbed NTCDA.

In conclusion, we applied the DFT-D3 method to obtain a very good description of the adsorption structure of NTCDA on the $\mathrm{Ag}(111)$ surface in comparison to experimental data. The environment-dependent dispersion coefficients in the DFT-D3 scheme seem to capture the main features of dispersion interactions in these systems.

(b) Vibrational analysis. On the basis of the excellent agreement of derived structural parameters with NIXSW experimental data, ${ }^{10}$ we have conducted a vibrational normal mode analysis for the structures obtained. In particular, such an analysis represents a direct link to the experiment. In order to obtain reference values for the various NTCDA normal modes, the structure and vibrations of free molecules (gas phase) were determined as well. For the latter system, investigations at the DFT level (PBE-D3 ${ }_{\mathrm{PBC}}$ ) could be compared to ab initio data from MP2/TZ calculations to evaluate the accuracy of the computations. The comparison of vibrational mode energies (Table 2) confirms the good agreement of the $\mathrm{PBE}-\mathrm{D} 3_{\mathrm{PBC}}$ results with the more accurate MP2 data. The RMS value for all frequencies in the range of $600-3200 \mathrm{~cm}^{-1}$ is $41 \mathrm{~cm}^{-1}$ without applying any scaling of the computed frequencies. This lends confidence to the usage of PBE-D3 ${ }_{\mathrm{PBC}}$ for the computation of the vibrational modes for NTCDA adsorbed on Ag(111), where a MP2 calculation is at the current stage not computationally feasible.

In Table 2 the individual vibrational modes of free and adsorbed NTCDA (bridge ${ }_{1}$ ) are listed. Also included are the irreducible representations of the various modes referring to free NTCDA $\left(D_{2 \mathrm{~h}}\right.$, see Scheme 1 for choice of coordinate system). We note that for the adsorbed molecule the symmetry can be $C_{2 \mathrm{v}}$ at most, i.e. for NTCDA occupying a high symmetry site. Various influences (effect of subsurface Ag-layer; lateral translations/rotations) may further reduce the symmetry to $C_{\mathrm{S}}$, $C_{2}$, or even $C_{1}$. According to Fig. 2, the deviations from $C_{2 \mathrm{v}}$ symmetry are minor.

In Table 2 the ordering and labelling of the individual modes has been taken from the free molecule. As interaction with the $\mathrm{Ag}(111)$ substrate affects the various modes differently, mode-crossings occur in a few cases which have been taken into account; from the inspection of individual displacement patterns of the molecular vibrations a direct correlation of modes for free and adsorbed NTCDA is easily obtained, though. All vibrational frequencies relevant to our experiment $\left(600-3200 \mathrm{~cm}^{-1}\right)$ are displayed - the full list of modes is available in Table S3 in the ESI $\dagger$ (Table S4 for all adsorption geometries investigated). Some of the vibrational modes are mainly located at specific functional groups. Due to their distinct 
Table 2 Computed vibrational modes $\left(>600 \mathrm{~cm}^{-1}\right.$ ) for molecular (free) NTCDA (MP2/TZ and PBE-D3 ${ }_{\text {PBC }}$ ) and comparison to values obtained for NTCDA adsorbed on Ag(111) (PBE-D3 PBC, $_{\text {, bridge }}$ site). Given are vibrational frequencies $\left(\nu\right.$, in $\left.\mathrm{cm}^{-1}\right)$ and IR-intensities ( $/$, normalized to the most intense band). The mode character is specified for selected modes only. A complete list of modes including the respective PBE-D3/TZ values is provided in the ESI (Table S3)

\begin{tabular}{|c|c|c|c|c|c|c|c|c|c|c|c|}
\hline \multirow{2}{*}{\multicolumn{2}{|c|}{ Mode $^{a}$}} & \multicolumn{4}{|c|}{ Free NTCDA molecule } & \multicolumn{5}{|c|}{ NTCDA/Ag(111) (bridge $_{1}$ ) } & \multirow[b]{3}{*}{ Mode character } \\
\hline & & \multicolumn{2}{|l|}{ MP2/TZ } & \multicolumn{2}{|c|}{$\underline{\mathrm{PBE}-\mathrm{D} 3_{\mathrm{PBC}}}$} & \multicolumn{5}{|c|}{$\underline{\text { PBE-D3 }}{ }_{\mathrm{PBC}}$} & \\
\hline \# & Symmetry & $\nu$ & $I$ & $\nu$ & $I$ & $\nu^{b}$ & $\nu^{c}$ & $I^{c}$ & $\nu_{\min }-\nu_{\max }^{d}$ & $\Delta \nu^{e}$ & \\
\hline 23 & $\mathrm{~b}_{1 \mathrm{u}}$ & 599.1 & 0.00 & 592.0 & 0.14 & 606.3 & 605.3 & 0.000 & 4.5 & 13.3 & \\
\hline 24 & $b_{3 g}$ & 600.5 & 0.00 & 593.5 & 0.00 & 602.8 & 603.1 & 0.000 & 3.9 & 9.6 & \\
\hline 25 & $a_{g}$ & 664.4 & 0.00 & 652.2 & 0.00 & 648.9 & 648.1 & 0.004 & 2.9 & -4.2 & \\
\hline 26 & $\mathrm{~b}_{2 \mathrm{u}}$ & 696.9 & 0.04 & 681.7 & 0.04 & 673.8 & 673.8 & 0.000 & 2.3 & -7.9 & \\
\hline 27 & $b_{2 g}$ & 727.6 & 0.00 & 686.9 & 0.00 & 665.4 & 665.4 & 0.000 & 7.1 & -21.5 & \\
\hline 28 & $b_{1 g}$ & 732.1 & 0.00 & 713.9 & 0.00 & 688.8 & 689.5 & 0.000 & 6.7 & -24.4 & \\
\hline 29 & $\mathrm{~b}_{3 \mathrm{u}}$ & 747.7 & 0.09 & 747.1 & 0.09 & 734.0 & 717.9 & 0.058 & 6.7 & -29.2 & \\
\hline 30 & $\mathrm{a}_{\mathrm{u}}$ & 751.5 & 0.00 & 753.9 & 0.00 & 720.7 & 720.8 & 0.000 & 6.5 & -33.1 & \\
\hline 31 & $\mathrm{~b}_{1 \mathrm{u}}$ & 752.2 & 0.00 & 745.3 & 0.07 & 750.7 & 748.8 & 0.000 & 4.8 & 3.5 & \\
\hline 32 & $\mathrm{~b}_{3 \mathrm{~g}}$ & 806.7 & 0.00 & 787.7 & 0.00 & 789.2 & 789.2 & 0.000 & 3.9 & 1.5 & \\
\hline 33 & $b_{1 u}$ & 828.6 & 0.00 & 812.4 & 0.05 & 796.0 & 813.6 & 0.000 & 4.6 & 1.2 & \\
\hline 34 & $b_{1 g}$ & 880.7 & 0.00 & 874.4 & 0.00 & 804.4 & 808.0 & 0.000 & 6.6 & -66.5 & \\
\hline 35 & $b_{3 u}$ & 881.9 & 0.05 & 874.6 & 0.04 & 815.8 & 813.0 & 0.002 & 5.4 & -61.6 & \\
\hline 36 & $\mathrm{a}_{\mathrm{u}}$ & 947.0 & 0.00 & 982.1 & 0.00 & 933.8 & 937.8 & 0.000 & 6.4 & -44.3 & \\
\hline 37 & $\mathrm{~b}_{2 \mathrm{u}}$ & 954.0 & 0.02 & 934.0 & 0.05 & 915.3 & 916.3 & 0.000 & 8.7 & -17.7 & \\
\hline 38 & $b_{2 g}$ & 961.4 & 0.00 & 982.3 & 0.00 & 939.1 & 943.3 & 0.000 & 7.0 & -39.0 & \\
\hline 39 & $\mathrm{a}_{\mathrm{g}}{ }^{\circ}$ & 1014.6 & 0.00 & 990.3 & 0.00 & 986.6 & 987.0 & 0.002 & 6.0 & -3.4 & \\
\hline 40 & $b_{3 g}$ & 1049.1 & 0.00 & 986.7 & 0.00 & 957.2 & 958.6 & 0.000 & 15.2 & -28.1 & $\mathrm{C}-\mathrm{O}_{\text {anh }}$ stretch \\
\hline 41 & $b_{2 u}$ & 1068.5 & 0.88 & 1011.6 & 0.79 & 958.0 & 959.5 & 0.000 & 11.6 & -52.1 & $\mathrm{C}-\mathrm{O}_{\text {anh }}$ stretch \\
\hline 42 & $a_{g}$ & 1125.0 & 0.00 & 1101.0 & 0.00 & 1101.0 & 1104.2 & 0.021 & 8.1 & 3.2 & \\
\hline 43 & $\mathrm{~b}_{1 \mathrm{u}}$ & 1135.2 & 0.35 & 1108.1 & 0.11 & 1088.1 & 1084.5 & 0.000 & 8.3 & -23.6 & \\
\hline 44 & $b_{3 g}$ & 1164.7 & 0.00 & 1139.9 & 0.00 & 1122.2 & 1123.9 & 0.000 & 7.2 & -16.0 & \\
\hline 45 & $\mathrm{~b}_{2 \mathrm{u}}$ & 1187.4 & 0.21 & 1156.5 & 0.13 & 1152.7 & 1155.8 & 0.000 & 7.2 & -0.7 & \\
\hline 46 & $b_{3 g}$ & 1235.8 & 0.00 & 1198.5 & 0.00 & 1207.2 & 1208.2 & 0.000 & 6.2 & 9.8 & \\
\hline 47 & $\mathrm{~b}_{2 \mathrm{u}}$ & 1280.9 & 0.27 & 1239.4 & 0.19 & 1210.4 & 1211.1 & 0.000 & 7.2 & -28.3 & \\
\hline 48 & $a_{g}$ & 1285.2 & 0.00 & 1248.2 & 0.00 & 1255.5 & 1256.9 & 0.038 & 6.7 & 8.7 & \\
\hline 49 & $\mathrm{~b}_{1 \mathrm{u}}$ & 1316.9 & 0.48 & 1283.9 & 0.50 & 1282.4 & 1282.5 & 0.000 & 7.1 & -1.3 & \\
\hline 50 & $a_{g}$ & 1392.2 & 0.00 & 1357.9 & 0.00 & 1344.5 & 1345.5 & 0.581 & 9.0 & -12.4 & \\
\hline 51 & $\mathrm{~b}_{1 \mathrm{u}}$ & 1475.3 & 0.04 & 1432.6 & 0.09 & 1437.8 & 1435.8 & 0.000 & 8.2 & 3.1 & \\
\hline 52 & $a_{g}$ & 1492.6 & 0.00 & 1429.0 & 0.00 & 1403.6 & 1404.8 & 0.133 & 13.7 & -24.1 & \\
\hline 53 & $b_{3 g}^{b}$ & 1498.6 & 0.00 & 1462.5 & 0.00 & 1419.5 & 1419.8 & 0.000 & 7.5 & -42.7 & \\
\hline 54 & $\mathrm{~b}_{2 \mathrm{u}}$ & 1527.5 & 0.00 & 1392.7 & 0.01 & 1429.2 & 1430.4 & 0.000 & 12.6 & 37.7 & \\
\hline 55 & $b_{2 u}$ & 1559.9 & 0.01 & 1519.5 & 0.02 & 1509.1 & 1509.8 & 0.000 & 9.7 & -9.7 & \\
\hline 56 & $\mathrm{~b}_{1 \mathrm{u}}$ & 1614.0 & 0.04 & 1575.8 & 0.07 & 1493.1 & 1493.6 & 0.000 & 12.5 & -82.2 & \\
\hline 57 & $\mathrm{a}_{\mathrm{g}}$ & 1628.6 & 0.00 & 1594.0 & 0.00 & 1564.7 & 1565.6 & 1.000 & 7.8 & -28.3 & \\
\hline 58 & $b_{3 g}$ & 1669.3 & 0.00 & 1617.8 & 0.00 & 1542.3 & 1541.2 & 0.000 & 12.5 & -76.6 & \\
\hline 59 & $\mathrm{~b}_{2 \mathrm{u}}$ & 1788.6 & 1.00 & 1733.1 & 1.00 & 1579.3 & 1580.9 & 0.000 & 21.6 & -152.2 & $\mathrm{C}=\mathrm{O} / \mathrm{C}=\mathrm{O}-\mathrm{Ag}$ \\
\hline 60 & $b_{3 g}$ & 1791.7 & 0.00 & 1734.0 & 0.00 & 1591.4 & 1593.0 & 0.000 & 29.2 & -141.0 & $\mathrm{C}=\mathrm{O} / \mathrm{C}=\mathrm{O}-\mathrm{Ag}$ \\
\hline 61 & $a_{g}$ & 1819.9 & 0.00 & 1770.6 & 0.25 & 1625.3 & 1625.7 & 0.264 & 22.1 & -144.9 & $\mathrm{C}=\mathrm{O} / \mathrm{C}=\mathrm{O}-\mathrm{Ag}$ \\
\hline 62 & $\mathrm{~b}_{1 \mathrm{u}}$ & 1824.1 & 0.73 & 1802.5 & 0.00 & 1655.7 & 1628.9 & 0.024 & 17.0 & -173.8 & $\mathrm{C}=\mathrm{O} / \mathrm{C}=\mathrm{O}-\mathrm{Ag}$ \\
\hline 63 & $\mathrm{~b}_{1 \mathrm{u}}$ & 3230.5 & 0.00 & 3114.3 & 0.00 & 3108.7 & 3112.2 & 0.000 & 5.1 & -2.1 & C-H stretch \\
\hline 64 & $b_{3 g}$ & 3230.7 & 0.00 & 3114.0 & 0.00 & 3109.1 & 3113.8 & 0.000 & 6.0 & -0.2 & C-H stretch \\
\hline 65 & $\mathrm{~b}_{2 \mathrm{u}}$ & 3242.4 & 0.01 & 3125.2 & 0.01 & 3120.9 & 3122.9 & 0.000 & 3.8 & -2.3 & C-H stretch \\
\hline 66 & $a_{g}$ & 3242.5 & 0.00 & 3125.1 & 0.00 & 3121.0 & 3126.5 & 0.000 & 6.5 & 1.5 & C-H stretch \\
\hline
\end{tabular}

${ }^{a}$ The sorting of modes and the symmetry assignment refers to the MP2/TZ data $\left(D_{2 \mathrm{~h}}\right.$ symmetry). Matching of the vibrational modes for the different levels of approximation has been carried out by visual inspection of the displacement pattern and comparison to MP2/TZ. ${ }^{b}$ Vibrational frequencies of adsorbed NTCDA computed with DFPT approach. ${ }^{c}$ Vibrational frequencies and IR intensities of adsorbed NTCDA computed with dynamical dipole approach. ${ }^{d}$ Spread of vibrational mode frequencies when considering all adsorption configurations for NTCDA/Ag. ${ }^{e}$ Change in vibrational frequency upon adsorption (relative to dynamical dipole-derived frequencies).

displacement patterns the four $\mathrm{C}-\mathrm{H}$ stretch modes, four $\mathrm{C}=\mathrm{O}$ stretch and two $\mathrm{C}-\mathrm{O}_{\text {anh }}$ stretch modes, and four $\mathrm{C}-\mathrm{H}$ bending modes can be readily assigned. For the most part, however, a clear assignment to a localized bond, or molecular sub-unit is not possible and the oscillation comprises characteristic motions (e.g. ring-breathing, ring-puckering) of the entire molecule. Detailed inspection identifies various deformation modes with specific polarizations, i.e. motion along the long or short axis of NTCDA, or perpendicular to the molecular plane.
The strongest shift in vibrational frequencies in going from the free to the adsorbed molecule $(\Delta \nu)$ can be found for the $\mathrm{C}=\mathrm{O}$ stretch modes due to the interaction with the $\mathrm{Ag}$ atoms at the surface. This shift in the range of $150 \mathrm{~cm}^{-1}$ is a strong indication for a directional interaction of the acyl oxygen atoms with Ag-atoms at the surface and in line with the conclusions drawn from the structural and charge-transfer data.

Another point to consider in the computation of the spectra is the possibility of the NTCDA molecule to occupy different 
binding sites on the $\operatorname{Ag}(111)$ surface as shown in Fig. 1. The simulation of infrared spectra for all binding geometries considered here leads to a range of frequencies for every normal mode given in Table $2\left(\nu_{\min }-\nu_{\max }\right)$. While the low frequency and the $\mathrm{C}-\mathrm{H}$ stretch modes display only small variations in vibrational frequencies, especially the modes connected to $\mathrm{C}-\mathrm{O}_{\mathrm{anh}}$ and $\mathrm{C}=\mathrm{O}$ stretching vibrations exhibit a spread of up to $30 \mathrm{~cm}^{-1}$. This is a clear indication that the directional interaction to the surface is somewhat dependent on the adsorption site occupied by NTCDA while the overall spectrum is only mildly influenced by the particular bonding configuration.

Comparison of calculated molecular vibrations with experimental IRAS data has to consider dipole selection rules. For molecules adsorbed on a flat metal substrate electric field components parallel to the surface plane are negligible and only vibrations with dynamic dipole moments perpendicular to the surface can be excited. For flat-lying, planar molecules (such as NTCDA on $\mathrm{Ag}(111)$ ) the surface plane is not a symmetry plane anymore and $D_{2 \mathrm{~h}}$ symmetry reduces to $C_{2 \mathrm{v}}$ as outlined above. This means that, in addition to the $b_{3 u}$ modes, all $a_{g}$ modes become dipole allowed as well. Further lowering of the adsorption site symmetry renders additional modes dipole active so that from an analysis of the IR spectra conclusions as to the local adsorption geometry can be drawn.

The computations of IR intensities for surface-adsorbed NTCDA were based upon a dynamical dipole approach considering the change of the dipole moment in $z$-direction only (see Fig. S1 in the ESI $\dagger$ ). Attempts to derive these intensities via the DFPT approach were not successful (results were strongly dependent on very minor changes in the adsorption geometry). As can be seen from Table 2, the vibrational frequencies derived with both approaches (denoted $\nu^{b}$ and $\nu^{c}$ ) are very similar. With exception of a few modes the differences are below $5 \mathrm{~cm}^{-1}$ $\left(\mathrm{RMS}=1.5 \mathrm{~cm}^{-1}\right)$.

\section{Experimental spectra}

In this section, the mode energies and IR intensities given in Table 2 are compared with the experimental spectrum. We note beforehand that NTCDA frequencies for the various adsorption configurations (Table S4 in the ESI $\dagger$ ) differ only slightly so that it is difficult to discriminate between different NTCDA sites.

In Fig. 6 the vibrational spectrum of a dilute layer of NTCDA/ $\mathrm{Ag}(111)$ is displayed. Specifically, 0.15 ML NTCDA has been deposited at $28 \mathrm{~K}$ and infrared absorption spectra taken right after transfer to our IR measurement cell. We note that thermal annealing (Fig. 6a) lead to slight but clearly discernible spectral changes which we attribute to lateral interactions among neighboring NTCDA. Initially, isolated NTCDA most likely occupying different sites should prevail. Upon successive annealing $(T \rightarrow 50 \mathrm{~K} \rightarrow 80 \mathrm{~K}$ ), NTCDA is expected to first transform into islands without long range order; eventually $(T \rightarrow 350 \mathrm{~K})$ islands exhibiting a distinct long range order (relaxed monolayer phase, see also Fig. S2 in the ESI $\dagger$ ) will form. These changes, however, will not be discussed in-depth here. The purpose to show these data is to prove that configurational alterations within the layer are in fact reflected in the
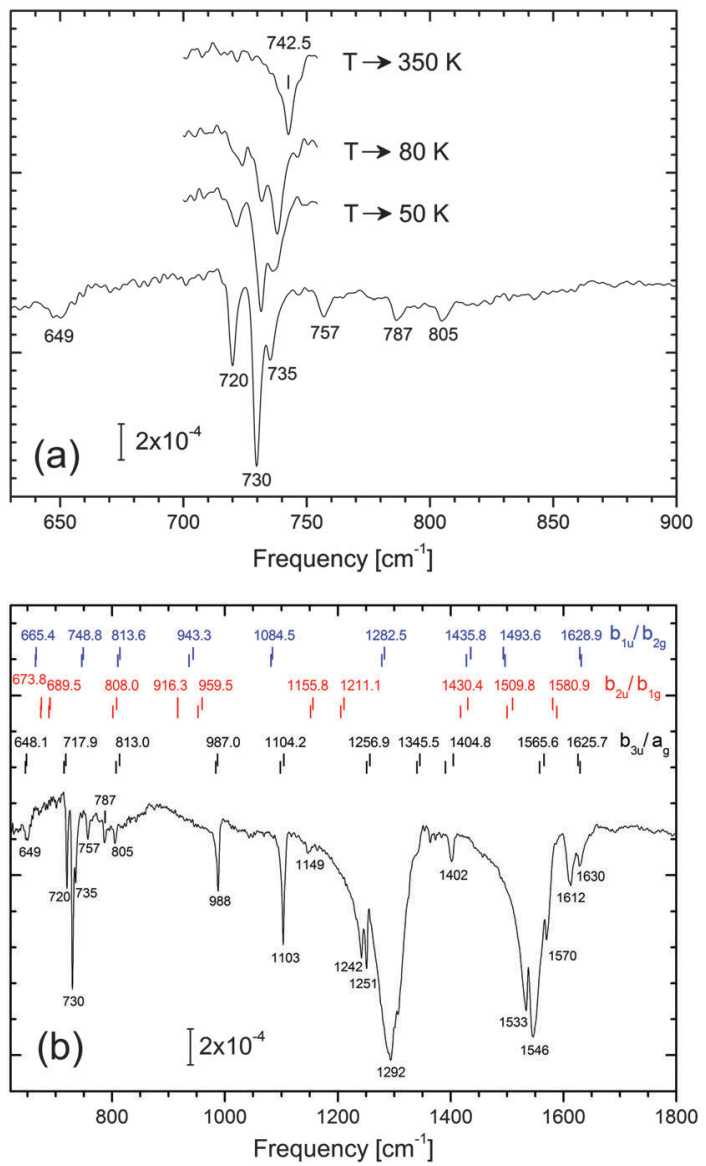

Fig. 6 IRAS spectra of $0.15 \mathrm{ML}$ NTCDA adsorbed on $\mathrm{Ag}(111)$ at $28 \mathrm{~K}$. (a) Frequency range $630-900 \mathrm{~cm}^{-1}$, emphasizing the prominent out-ofplane mode at about $730 \mathrm{~cm}^{-1}$. In panel (a) the thermal evolution of this mode is displayed; the spectra refer to the layer after annealing to the indicated temperatures. (b) Frequency range $620-1800 \mathrm{~cm}^{-1}$, featuring predominantly in-plane (ip) modes of NTCDA at $>900 \mathrm{~cm}^{-1}$. The calculated line positions (PBE-D3 ${ }_{\mathrm{PBC}}$, dynamic dipole approach) are indicated by vertical lines and labelled according to their vibrational frequencies. Modes of different symmetry properties are grouped in separate rows: $b_{3 u}$ and $a_{g}$ (black); $b_{2 u}$ and $b_{1 g}$ (red); $b_{1 u}$ and $b_{2 g}$ (blue). The two bars for each set of modes refer to values of NTCDA occupying bridge $e_{1}$ and top $_{1}$ sites. All spectra were obtained at $28 \mathrm{~K}$ and $2 \mathrm{~cm}^{-1}$ spectral resolution.

observed vibrational spectra and can be traced sensitively. Most relevant for our comparison to the calculated spectra is that the low NTCDA coverage (0.15 ML) ensures that isolated NTCDA are present and intermolecular interactions are negligible. This assumption is corroborated by similar data sets obtained at 0.1 and 0.3 ML (not shown). Specifically, virtually identical modes are observed at $0.1 \mathrm{ML}$, while at coverages of $0.3 \mathrm{ML}$ intermolecular interactions start becoming relevant. We note that the spectrum displayed in Fig. $6 \mathrm{~b}$ represents the sum of several consecutively taken spectra and within this series no alterations in vibrational peak positions and intensities have been found.

In Fig. $6 \mathrm{~b}$ we have included the calculated PBE-D3 $3_{\mathrm{PBC}}$ line positions of the various modes (dynamical dipole approach) and sorted them according to their symmetry. In order to avoid ambiguities, we kept the original nomenclature of the $D_{2 \mathrm{~h}}$ 
symmetry group. The bottom row (black) contains modes which should be dipole active even if a high symmetry site $\left(C_{2 \mathrm{v}}\right.$ symmetry) is occupied, e.g. modes belonging to the $\mathrm{b}_{3 \mathrm{u}}$ and $\mathrm{a}_{\mathrm{g}}$ irreducible representations of the $D_{2 \mathrm{~h}}$ symmetry group. In case that the molecule is laterally displaced in the $\mathrm{O}-\mathrm{O}$ direction $b_{1 \mathrm{u}}$ and $b_{2 \mathrm{~g}}$ type of modes become dipole active as well (symmetry reduces to $C_{\mathrm{S}}$ ); similarly, the inequality of fcc and hcp hollow sites (or some other symmetry-breaking element in the $y$-direction) would lead to $C_{\mathrm{S}}$ symmetry at most so that $\mathrm{b}_{2 \mathrm{u}}$ as well as $b_{1 \mathrm{~g}}$ type of modes should display some intensity in IR spectra. The additional modes expected in the IR spectra are listed in the top and middle rows of Fig. 6b, respectively. Yet another type of displacement from a high symmetry site would be an azimuthal rotation or warping of the molecular plane (rendering $\mathrm{b}_{3 \mathrm{~g}}$ and $\mathrm{a}_{\mathrm{u}}$ type of modes dipole active), but based on the computed adsorption energies, these displacements are energetically unfavourable.

We will now turn to the $700-1200 \mathrm{~cm}^{-1}$ region and analyse whether the observed spectral features are in line with a symmetry-reduced adsorption geometry. From a brief inspection of the spectrum in Fig. $6 b$ the $b_{3 u}$ and $a_{g}$ modes are readily identified. As the observed spectrum contains no significant additional modes we conclude that deviations from the local $C_{2 \mathrm{v}}$ symmetry are only weak. In particular, the absence of modes at 915, 960 and $1085 \mathrm{~cm}^{-1}$ lead us to conclude that such a symmetry-breaking element is missing (or its effect is negligible). On the other hand, we clearly observe a band at $1149 \mathrm{~cm}^{-1}$ which cannot be associated with $\mathrm{a}_{\mathrm{g}}, \mathrm{b}_{3 \mathrm{u}}, \mathrm{b}_{1 \mathrm{u}}$, or $\mathrm{b}_{2 \mathrm{~g}}$ modes.

According to the fine structure of modes at 720-735, 1103, $1242 / 1251,1533-1546,1612-1630 \mathrm{~cm}^{-1}$ we invoke that more than just a single adsorption site is occupied. Moreover, structural disorder resulting from the low NTCDA deposition temperature may contribute to the heterogeneous line shapes or the presence of weak extra bands. The spectral regions around $1290 \mathrm{~cm}^{-1}$ and $1550 \mathrm{~cm}^{-1}$ are particularly crowded and, even though tiny details of the respective broad absorption bands are highly reproducible, their detailed assignment remains vague at present; we will merely list some ideas as to their origin: (i) thermally excited low frequency modes (e.g. translations/rotations parallel to the surface) lead to broadening of vibrational modes by means of anharmonic coupling. (ii) In addition to existing fundamental modes of adsorbed NTCDA, combination bands additionally contribute; by means of Fermi resonance coupling to spectrally close fundamental modes, ${ }^{30,79}$ these weak bands could gain substantial in intensity. (iii) Breaking of the local adsorption site symmetry adds new modes. (iv) Finally, a factor contributing to heterogeneity in vibrational spectra refers to slight line shifts due to naturally abundant ${ }^{13} \mathrm{C}(1.1 \%)$ located at random positions within the carbon skeleton of NTCDA.

According to Fig. 6, vibrational bands which appear well suited for a detailed analysis are the $\mathrm{C}-\mathrm{O}$ stretch modes of the acyl group at $1600-1800 \mathrm{~cm}^{-1}$, besides the spectral region at $700-1200 \mathrm{~cm}^{-1}$. The $\mathrm{C}-\mathrm{H}$ stretching modes at $3000-3200 \mathrm{~cm}^{-1}$, on the other hand, are too weak to be detected and are therefore omitted in Fig. 6. In addition to their generally small dynamic dipole moments, the parallel orientation of adsorbed NTCDA further aggravates their detection. At 1612 and $1630 \mathrm{~cm}^{-1}$ we observe two distinct acyl $\mathrm{C}-\mathrm{O}$ stretch modes. Due to their high frequencies and the low density of modes in this spectral region there is little doubt regarding their assignment. At $1600-1650 \mathrm{~cm}^{-1}$ the acyl group modes appear at substantially lower frequencies as compared to gas phase or bulk NTCDA for which $150-200 \mathrm{~cm}^{-1}$ higher frequencies are found (Table 2). We conclude that the oxygen atoms interact strongly with underlying Ag substrate atoms. As a consequence of this chemical interaction, a weakening of the $\mathrm{C}-\mathrm{O}$ bond as well as a bending of the $\mathrm{CO}$ unit towards the surface occurs. This is in line with the outcome of our theoretical analysis and NIXSW findings. ${ }^{9,10}$

For NTCDA adsorbed on a high symmetry site $\left(C_{2 \mathrm{v}}\right.$ symmetry) only one $\mathrm{C}=\mathrm{O}$ stretch mode is expected which can be ascribed to the symmetric stretching of the $\mathrm{C}-\mathrm{O}$ bonds $\left(\mathrm{a}_{\mathrm{g}}\right)$ of isolated NTCDA on $\mathrm{Ag}(111)$. The question therefore arises as to the origin of the second mode. Three explanations appear reasonable: (i) two different high symmetry sites are occupied and the low sample temperature prevents migration from the less strongly bound to the energetically preferred site. (ii) NTCDA occupies a single site only which, however, is not a high symmetry site, e.g. the molecule is laterally displaced or rotated. (iii) the $1630 \mathrm{~cm}^{-1}$ band may indicate the formation of NTCDA dimers or small aggregates; in this respect we mention that for a dense layer the acyl band displays a notable blue shift leading to a broad band at $1625-1675 \mathrm{~cm}^{-1}$ (see Fig. S2, ESI $\dagger$ ).

\section{Conclusions}

By combining $a b$ initio computations with high-resolution IRAS measurements an in-depth understanding of molecular layers on metal surfaces has been attained for the model system NTCDA on Ag(111). From the excellent agreement between calculated and experimentally found molecular structure data, as well as molecular vibrations, we conclude that DFT including dispersion forces is ideally suited to characterize organic (mono)layer films involving large molecules. For these systems van der Waals interactions and chemical bonding are equally important and omitting either one leads to erroneous results. Common flaws, such as much too weak bonding or an incorrect molecular structure are effectively precluded. In general, the reproduction of structural parameters as derived from NIXSW experiments is very good, especially regarding the overall bonding distance and a downward bending of the acyl groups. Slight deviations between calculated and experimental structural data are attributed to the effect of lateral interactions among neighbouring NTCDA molecules.

We find a directional interaction between the acyl oxygen atoms of NTCDA and specific Ag atoms of the Ag(111) surface. This is supported by a notable corrugation of the $\mathrm{Ag}$ surface, the dependence of adsorption energies on the local adsorption configurations (e.g. with and without short $\mathrm{Ag}-\mathrm{O}_{\mathrm{acyl}}$ distances), specific frequency shifts upon adsorption and structural changes 
in the NTCDA molecule. As to the origin of these latter modifications we confirm a downshift and partial filling of the formerly unoccupied NTCDA LUMO. While similar structural and electronic changes upon adsorption have very recently been reported for PTCDA/Ag(111), ${ }^{3}$ quantitative comparison of computed and experimental vibrational spectra is provided for the first time for such a large organic molecule adsorbed on a metal surface. The high accuracy of the calculation in reproducing the experimental frequency values across the full IR frequency range, leads us to conclude that errors should be only minor, at least in spectral regions with low or only moderately high mode densities.

\section{Acknowledgements}

RT thanks Jonas Möllmann and Stefan Grimme (Bonn) for providing their development version of the DFT-D3 code, Peter Schwerdtfeger (Auckland) for helpful discussions and HLR Stuttgart, CSC Frankfurt (LOEWE), HRZ Marburg and HLR Stuttgart for providing computational resources. We thank Carolin R. Braatz and Gregor Öhl for technical assistance during experiments. Financial support is provided by the DFG via the SFB 1083 "Structure and Dynamics of Internal Interfaces" and GRK 1782 "Functionalization of Semiconductors".

\section{Notes and references}

1 J. V. Barth, Annu. Rev. Phys. Chem., 2007, 58, 375.

2 N. Ueno and S. Kera, Prog. Surf. Sci., 2008, 83, 490.

3 O. Bauer, G. Mercurio, M. Willenbockel, W. Reckien, C. H. Schmitz, B. Fiedler, S. Soubatch, T. Bredow, F. S. Tautz and M. Sokolowski, Phys. Rev. B: Condens. Matter Mater. Phys., 2012, 86, 235431.

4 U. Stahl, D. Gador, A. Soukopp, R. Fink and E. Umbach, Surf. Sci., 1998, 414, 423.

5 L. Kilian, U. Stahl, I. Kossev, M. Sokolowski, R. Fink and E. Umbach, Surf. Sci., 2008, 602, 2427.

6 D. Gador, C. Buchberger, R. Fink and E. Umbach, Europhys. Lett., 1998, 41, 231.

7 A. Bendounan, F. Forster, A. Schöll, D. Batchelor, J. Ziroff, E. Umbach and F. Reinert, Surf. Sci., 2007, 601, 4013.

8 A. Schöll, Y. Zou, L. Kilian, D. Hübner, D. Gador, C. Jung, S. G. Urquhart, T. Schmidt, R. Fink and E. Umbach, Phys. Rev. Lett., 2004, 93, 146406.

9 J. Stanzel, W. Weigand, L. Kilian, H. L. Meyerheim, C. Kumpf and E. Umbach, Surf. Sci., 2004, 571, L311.

10 C. Stadler, S. Hansen, A. Schöll, T.-L. Lee, J. Zegenhagen, C. Kumpf and E. Umbach, New J. Phys., 2007, 9, 50.

11 J. Hafner, J. Comput. Chem., 2008, 29, 2044.

12 P. J. Hasnip, K. Refson, M. I. J. Probert, J. R. Yates, S. J. Clark and C. J. Pickard, Philos. Trans. R. Soc., A, 2014, 372, 26.

13 W. Liu, A. Tkatchenko and M. Scheffler, Acc. Chem. Res., 2014, 47, 3369.

14 K. Burke, J. Chem. Phys., 2012, 136, 150901.

15 S. Grimme, Wiley Interdiscip. Rev.: Comput. Mol. Sci., 2011, 1, 211.
16 A. Tkatchenko, L. Romaner, O. T. Hofmann, E. Zojer, C. Ambrosch-Draxl and M. Scheffler, MRS Bull., 2010, 35, 435.

17 J. Grafenstein and D. Cremer, J. Chem. Phys., 2009, 130, 124105.

18 E. R. Johnson, I. D. Mackie and G. A. DiLabio, J. Phys. Org. Chem., 2009, 22, 1127.

19 T. Sato and H. Nakai, J. Chem. Phys., 2009, 131, 224104.

20 S. Grimme, D. Heitmann, T. Pape, A. Hepp, C. Mück-Lichtenfeld and F. E. Hahn, J. Am. Chem. Soc., 2011, 133, 11118.

21 L. Goerigk, H. Kruse and S. Grimme, ChemPhysChem, 2011, 12, 3421.

22 W. Reckien and T. Bredow, Chem. Phys. Lett., 2011, 508, 54.

23 J. Moellmann and S. Grimme, Phys. Chem. Chem. Phys., 2010, 12, 8500.

24 T. Bučko, J. Hafner, S. Lebegue and J. G. Angyán, J. Phys. Chem. A, 2010, 114, 11814.

25 L. Maschio, B. Civalleri, P. Ugliengo and A. Gavezzotti, J. Phys. Chem. A, 2011, 115, 11179.

26 J. Moellmann, S. Ehrlich, R. Tonner and S. Grimme, J. Phys.: Condens. Matter, 2012, 24, 424206.

27 V. G. Ruiz, W. Liu, E. Zojer, M. Scheffler and A. Tkatchenko, Phys. Rev. Lett., 2012, 108, 146103.

28 M. Eremtchenko, D. Bauer, J. A. Schaefer and F. S. Tautz, New J. Phys., 2004, 6, 4.

29 F. S. Tautz, S. Sloboshanin, J. A. Schaefer, R. Scholz, V. Shklover, M. Sokolowski and E. Umbach, Phys. Rev. B: Condens. Matter Mater. Phys., 2000, 61, 16933.

30 T. Breuer, M. A. Celik, P. Jakob, R. Tonner and G. Witte, J. Phys. Chem. C, 2012, 116, 14491.

31 C. R. Braatz, G. Öhl and P. Jakob, J. Chem. Phys., 2012, 136, 134706.

32 C. R. Braatz, T. Esat, C. Wagner, R. Temirov, F. S. Tautz and P. Jakob, Surf. Sci., 2016, 643, 98.

33 F. S. Tautz, Prog. Surf. Sci., 2007, 82, 479.

34 R. Temirov, S. Soubatch, A. Lassise and F. S. Tautz, J. Phys.: Condens. Matter, 2008, 20, 224010.

35 F. S. Tautz, M. Eremtchenko, J. A. Schaefer, M. Sokolowski, V. Shklover and E. Umbach, Phys. Rev. B: Condens. Matter Mater. Phys., 2002, 65, 125405.

36 P. Rosenow, P. Jakob, R. Tonner, submitted.

37 M. J. Frisch, G. W. Trucks, H. B. Schlegel, G. E. Scuseria, M. A. Robb, J. R. Cheeseman, G. Scalmani, V. Barone, B. Mennucci, G. A. Petersson, H. Nakatsuji, M. Caricato, X. Li, H. P. Hratchian, A. F. Izmaylov, J. Bloino, G. Zheng and J. L. H. Sonnenberg, M. Ehara, K. Toyota, R. Fukuda, J. Hasegawa, M. Ishida, T. Nakajima, Y. Honda, O. Kitao, H. Nakai, T. Vreven, J. A. Montgomery, Jr., J. E. Peralta, F. Ogliaro, M. Bearpark, J. J. Heyd, E. Brothers, K. N. Kudin, V. N. Staroverov, R. Kobayashi, J. Normand, K. Raghavachari, A. Rendell, J. C. Burant, S. S. Iyengar, J. Tomasi, M. Cossi, N. Rega, N. J. Millam, M. Klene, J. E. Knox, J. B. Cross, V. Bakken, C. Adamo, J. Jaramillo, R. Gomperts, R. E. Stratmann, O. Yazyev, A. J. Austin, R. Cammi, C. Pomelli, J. W. Ochterski, R. L. Martin, K. Morokuma, V. G. Zakrzewski, G. A. Voth, P. Salvador, J. J. Dannenberg, S. Dapprich, A. D. Daniels, Ö. Farkas, J. B. Foresman, J. V. Ortiz, J. Cioslowski and D. J. Fox, Gaussian, Inc., Wallingford CT, 2009. 
38 R. Ahlrichs, M. Bär, M. Häser, H. Horn and C. Kölmel, Chem. Phys. Lett., 1989, 162, 165.

39 J. P. Perdew, K. Burke and M. Ernzerhof, Phys. Rev. Lett., 1996, 77, 3865.

40 F. Weigend, Phys. Chem. Chem. Phys., 2006, 8, 1057.

41 S. Grimme, J. Antony, S. Ehrlich and H. Krieg, J. Chem. Phys., 2010, 132, 154104.

42 S. Grimme, S. Ehrlich and L. Goerigk, J. Comput. Chem., 2011, 32, 1456.

43 P. Deglmann, F. Furche and R. Ahlrichs, Chem. Phys. Lett., 2002, 362, 511.

44 K. Eichkorn, O. Treutler, H. Ohm, M. Häser and R. Ahlrichs, Chem. Phys. Lett., 1995, 240, 283.

45 G. Kresse and J. Furthmüller, Phys. Rev. B: Condens. Matter Mater. Phys., 1996, 54, 11169.

46 P. E. Blöchl, Phys. Rev. B: Condens. Matter Mater. Phys., 1994, 50, 17953.

47 G. Kresse and D. Joubert, Phys. Rev. B: Condens. Matter Mater. Phys., 1999, 59, 1758.

48 L. Romaner, D. Nabok, P. Puschnig, E. Zojer and C. Ambrosch-Draxl, New J. Phys., 2009, 11, 053010.

49 M. C. E. Galbraith, M. Marks, R. Tonner and U. Höfer, J. Phys. Chem. Lett., 2014, 5, 50.

50 S. S. Tsirkin, N. L. Zaitsev, I. A. Nechaev, R. Tonner, U. Höfer and E. V. Chulkov, Phys. Rev. B: Condens. Matter Mater. Phys., 2015, 92, 235434.

51 H. J. Monkhorst and J. D. Pack, Phys. Rev. B: Solid State, 1976, 13, 5188.

52 P. Giannozzi and S. Baroni, J. Chem. Phys., 1994, 100, 8537.

53 S. Baroni, S. de Gironcoli, A. Dal Corso and P. Giannozzi, Rev. Mod. Phys., 2001, 73, 515.

54 M. Gajdoš, K. Hummer, G. Kresse, J. Furthmüller and F. Bechstedt, Phys. Rev. B: Condens. Matter Mater. Phys., 2006, 73, 045112.

55 S. Grimme, J. Comput. Chem., 2006, 27, 1787.

56 E. Sanville, S. D. Kenny, R. Smith and G. Henkelman, J. Comput. Chem., 2007, 28, 899.

57 R. Dronskowski and P. E. Blöchl, J. Phys. Chem., 1993, 97, 8617.

58 V. L. Deringer, A. L. Tchougréeff and R. Dronskowski, J. Phys. Chem. A, 2011, 115, 5461.
59 S. Maintz, V. L. Deringer, A. L. Tchougréeff and R. Dronskowski, J. Comput. Chem., 2013, 34, 2557.

60 C. Bunge, J. Barrientos and A. Bunge, At. Data Nucl. Data Tables, 1993, 53, 113.

61 A. Tkatchenko, R. A. DiStasio, R. Car and M. Scheffler, Phys. Rev. Lett., 2012, 108, 236402.

62 E. A. Soares, G. S. Leatherman, R. D. Diehl and M. A. Van Hove, Surf. Sci., 2000, 468, 129.

63 C. E. Botez, W. C. Elliott, P. F. Miceli and P. W. Stephens, Phys. Rev. B: Condens. Matter Mater. Phys., 2001, 63, 113404.

64 J. J. Xie, S. de Gironcoli, S. Baroni and M. Scheffler, Phys. Rev. B: Condens. Matter Mater. Phys., 1999, 59, 970.

65 A. Hauschild, K. Karki, B. C. C. Cowie, M. Rohlfing, F. S. Tautz and M. Sokolowski, Phys. Rev. Lett., 2005, 94, 036106.

66 A. Hauschild, R. Temirov, S. Soubatch, O. Bauer, A. Schöll, B. C. C. Cowie, T.-L. Lee, F. S. Tautz and M. Sokolowski, Phys. Rev. B: Condens. Matter Mater. Phys., 2010, 81, 125432.

67 C. Kumpf, private communication.

68 A. Alkauskas, A. Baratoff and C. Bruder, Phys. Rev. B: Condens. Matter Mater. Phys., 2006, 73, 165408.

69 L. Born and G. Heywang, Z. Kristallogr., 1990, 190, 147.

70 J. Ziroff, F. Forster, A. Schöll, P. Puschnig and F. Reinert, Phys. Rev. Lett., 2010, 104, 233004.

71 L. Kilian, A. Hauschild, R. Temirov, S. Soubatch, A. Schöll, A. Bendounan, F. Reinert, T.-L. Lee, F. S. Tautz, M. Sokolowski and E. Umbach, Phys. Rev. Lett., 2008, 100, 136103.

72 E. R. McNellis, J. Meyer and K. Reuter, Phys. Rev. B: Condens. Matter Mater. Phys., 2009, 80, 205414.

73 F. H. Allen, Acta Crystallogr., 2002, B58, 380.

74 A. Hauschild, K. Karki, B. C. C. Cowie, M. Rohlfing, F. S. Tautz and M. Sokolowski, Phys. Rev. Lett., 2005, 95, 209602.

75 K. A. Fichthorn and R. A. Miron, Phys. Rev. Lett., 2002, 89, 196103.

76 R. Zacharia, H. Ulbricht and T. Hertel, Phys. Rev. B: Condens. Matter Mater. Phys., 2004, 69, 155406.

77 D. Käfer, C. Wöll and G. Witte, Appl. Phys. A: Mater. Sci. Process., 2009, 95, 273.

78 M. Rohlfing, R. Temirov and F. S. Tautz, Phys. Rev. B: Condens. Matter Mater. Phys., 2007, 76, 115421.

79 P. Jakob, J. Chem. Phys., 1998, 108, 5035. 